\title{
The Aviation Anti-Submarine Warfare Expert in Military Conceptual Model Based on AHP Confirm Method Research
}

\author{
Yangyang Zhao ${ }^{1}$ a Liang $^{M^{2 b}}$ and Xiaoxiao Yang ${ }^{3 \mathrm{c}}$ \\ ${ }^{1}$ Naval submarine Academy of simulator training center, Dalian, 116025, China; \\ ${ }^{2}$ Department of Surface Ship Command, Dalian Naval Academy, Dalian, 116018, China; \\ ${ }^{3}$ Naval submarine Academy, Qingdao, 266022, China; \\ 285892287@qq.com, maliang2014@tom.com,15953250486@163.com
}

Keywords: AHP; Aviation anti-submarine warfare; Military conceptual model; Confirm the method

\begin{abstract}
Military conceptual model is the first layer of abstraction of real world military knowledge, it is the foundation of simulation system is established, and is an important method of the whole simulation system VV\&A. This article is based on the AHP method to the aviation anti-submarine warfare, military conceptual model of expert validation method is studied.
\end{abstract}

\section{Introduction}

Aircraft taking off from the carrier, to complete the mission, the ship and transferred to the hangar and maintenance of this a series of actions involving numerous aircraft carrier aircraft security resources, how to rapid distribution security resources rationally, to more than aircraft security safeguards the rational planning of process, process, when the relationship between the combat effectiveness of aircraft carrier aircraft.

Military conceptual model is the first layer of abstraction of real world military knowledge, it is the foundation of simulation system is established, and is an important method of the whole simulation system VV\&A. At the same time, the military conceptual modeling can improve the reuse of domain knowledge and authority, is the foundation of simulation system interoperability. This article is based on the AHP method to the aviation anti-submarine warfare, military conceptual model of expert validation method is studied.

\section{The Quality Problem of the Military Conceptual Model}

We know that the military conceptual model is an important part of the simulation system, but the military conceptual model is good or bad, right or not directly related to the simulation system is good or bad. good quality model, will eventually get users and experts confirmed that quality is bad model will not be confirmed. The same about the aviation anti-submarine warfare, military conceptual model.

Military conceptual model for the aviation anti-submarine warfare experts confirmed indicators are necessity, consistency, correctness, completeness, clarity, and so on.

Validity: aviation anti-submarine military conceptual model must be the same with the actual circumstances of the aviation anti-submarine warfare to, described in the content must be accurate, not inconsistent with the objective facts, otherwise it's meaningless to the military conceptual model.

Integrity: aviation anti-submarine military conceptual model must cover all parts of the aviation anti-submarine warfare. If neglect important operational process, said that the conceptual model does not meet the integrity.

Necessity: aviation anti-submarine military conceptual model describes the process must be concise, seize the key part of the aviation anti-submarine warfare, try to avoid irrelevant, spare parts, don't be too complicated, can't let the simulation technicians are difficult to read and understand. 
Consistency: the consistency of the aviation anti-submarine warfare, military conceptual model is refers to between each part must be consistent with each other, can't appear some inconsistent with the rest of the content.

Clarity: aviation anti-submarine military conceptual model of all parts must be clear, not ambiguous, affect the understanding of the model. [3]

\section{Aviation Antisubmarine Assessment of Military Conceptual Model}

For military conceptual model of evaluation index, the aviation anti-submarine warfare military conceptual model is to evaluate from the aspects of building model of the steps.

the aviation anti-submarine warfare military conceptual model is the important step is to build a concept model of resources. Entity, action, task and interaction are the four basic modeling elements of military conceptual model, the conceptual model of four basic resources.

"Correctness" of model resources main requirements are: entity, action, task and interaction of expression should be accurate; Entity mainly include antisubmarine plane, the enemy submarines, acoustic buoy, sonar, torpedoes, etc., action mainly entity in carrying out a series of behaviors of aviation anti-submarine warfare, such as antisubmarine warfare aircraft takeoff, mobile, have to evade, turn back, the main task is the purpose of reflects the behavior of entities, such as the task of antisubmarine warfare aircraft is anti-submarine mission is to perform a certain sea area, the enemy submarines patrol, attack, etc., the interaction is the exchange of information between entities, antisubmarine warfare aircraft and the command post of information and communication, etc., to these resources must be to describe them as required, to conform to the description form, it can reflect the correctness of the model of resources.

Aviation anti-submarine military conceptual model model of resource requirement is "integrity", to each entity, action, task and interaction of statement to include all the parts, such as physical manipulation of the commander, driver, armament, surface ships, anti-submarine helicopters, fixed wing antisubmarine warfare aircraft, dipping sonar and sonobuoy, torpedo, depth bomb, fire control system, base, detection information and instructions. Other actions, tasks, interaction is complete.

Aviation anti-submarine military conceptual model model resources, is the "necessity" demand of resources in constructing aviation anti-submarine military conceptual model of "integrity" at the same time, must embody the "necessity" of model resources, seize the key part of the aviation anti-submarine warfare, try to avoid irrelevant and redundant description. Many entity has nothing to do with the aviation anti-submarine warfare, action, task and interaction doesn't have to, such as antisubmarine warfare aircraft movements and possibly alert to empty, this is has nothing to do with the anti-submarine warfare, is unnecessary in the build action resources fully.

Aviation anti-submarine military conceptual model model resources, are the "consistency" requirements for each entity, action, task and interaction be consistent to the description of the aviation anti-submarine warfare, which is a description of the various attributes of the entity, action, task description, the description of the interaction part should be consistent with each other, can not be described earlier resources and the resources behind the inconsistencies. Such as physical attributes of the task description is the parent of task, task description of resources are not subtasks.

Aviation anti-submarine military conceptual model model resources, are the "clarity" requirements for each entity, action, task and interaction description language to describe, clear form to meet the requirements, and can't let technical personnel understand the ambiguity. As in the construction entity, antisubmarine plane this entity would probably be divided into anti-submarine helicopters and fixed-wing antisubmarine warfare aircraft; the clear and clear.

aviation anti-submarine military conceptual model is another important step is the description of the operational rules. Action description is to describe elements give detailed rules process and processing rules. Rules describe mainly includes: antisubmarine warfare aircraft take-off condition, flight rules, determine the search equipment rules, use the search rules, height of search, search speed, tracking, rules, and determine against rules, the use of weapons and weapons delivery rules, avoid and its rules, logical description and so on. These rules must carry on the detailed narration to the simulation technicians to a better understanding of operations 
"Correct" description of operational rules, the aviation anti-submarine operational rules of military conceptual model of each description must be authoritative, conform to the rules of the aviation anti-submarine warfare. Such as antisubmarine warfare aircraft take-off condition rule to the wind velocity, visibility, cloud height and so on, if the does not conform to that stipulated in the actual terms of antisubmarine warfare aircraft take-off or inappropriate, is considered not correct.

Aviation anti-submarine operational rules of military conceptual model described in the "integrity" and "necessity" and "consistency", "clarity" requirement, respectively is: "integrity" is a description of the aviation anti-submarine operational rules of military conceptual model is comprehensive, complete and not missing or forgotten some rules of anti-submarine warfare; "Necessity" is refers to the description of the rules to seize a key part of the aviation anti-submarine warfare as far as possible; "Consistency" is the description of the rules should be consistent with each other; "Clarity" refers to the description language is clear and easy to read.

The design of the aviation anti-submarine warfare, military conceptual model, and description of the process of operation, data requirements, operational background and so on. Their evaluation with the first two are from necessity, consistency, correctness, completeness, clarity.

\section{Acknowledging the Aviation Anti-Submarine Warfare, Military Conceptual Model Based on AHP Method}

Aviation Antisubmarine Assessment of Military Conceptual Model Hierarchy. In the application of AHP method to confirm the analysis, the first to streamline the problem of aviation anti-submarine military conceptual model, hierarchical, and construct a hierarchical structure model, the aviation anti-submarine military conceptual model of evaluation hierarchical structure. At the highest levels, that is, target layer, it should be the goal of the problem or as a result, the ideal for aviation anti-submarine military conceptual model, it should be a model of total confirmed; Rule of the middle layer is layer contains the goals involved in the intermediate links, for aviation anti-submarine military conceptual model, it should be all aspects of the implementation model of total confirmed degree, we can with the aviation anti-submarine build steps of military conceptual model (as described before) to assess. The middle layer is divided into resources, operational rules model description, description, the process of operation data requirements, other processes, etc., were expressed in $C_{1} 、 C_{2} 、 C_{3} 、 C_{4} 、 C_{5}$, Also known as the bottom layer or solution measure, the aviation anti-submarine warfare military conceptual model is fully available for evaluation, basic use, part available, unavailable, expressed in $S_{1} 、 S_{2} 、 S_{3} 、 S_{4}$ respectively. Hierarchical structure below.

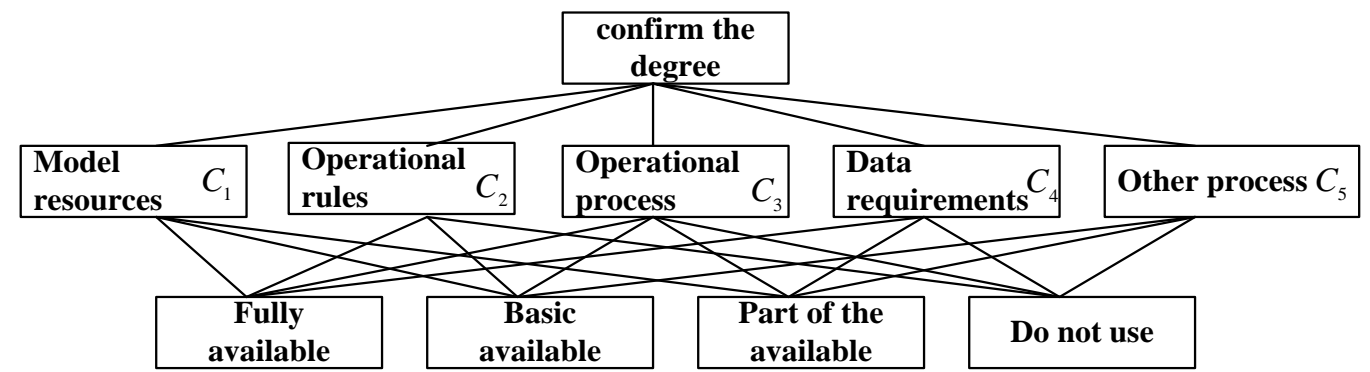

Figure 1. Aviation anti-submarine military conceptual model evaluation hierarchical structure

Model Assessment and Consistency Check Of Judgment Matrix. According to the aviation anti-submarine warfare, military conceptual model to evaluate the level of structure to construct the rule layer, that is, to build the model of resources and operational rules of description, describes the process of operation, data requirements, relative to other process always confirm the degree of judgment matrix, consistency and calculate each factor weight finally. Judgment matrix is as follows: 
Table 1 judgment matrix

\begin{tabular}{|c|c|c|c|c|c|}
\hline Confirm degree & $C_{1}$ & $C_{2}$ & $C_{3}$ & $C_{4}$ & $C_{5}$ \\
\hline$C_{1}$ & $c_{11}$ & $c_{12}$ & $c_{13}$ & $c_{14}$ & $c_{15}$ \\
\hline$C_{2}$ & $c_{21}$ & $c_{22}$ & $c_{23}$ & $c_{24}$ & $c_{25}$ \\
\hline$C_{3}$ & $c_{31}$ & $c_{32}$ & $c_{33}$ & $c_{34}$ & $c_{35}$ \\
\hline$C_{4}$ & $c_{41}$ & $c_{42}$ & $c_{43}$ & $c_{44}$ & $c_{45}$ \\
\hline$C_{5}$ & $c_{51}$ & $c_{52}$ & $c_{53}$ & $c_{54}$ & $c_{55}$ \\
\hline
\end{tabular}

$c_{11}, c_{22}, c_{33}, c_{44}, c_{55}=1, \quad c_{i j}=1 / c_{j i}$ 。

Computing model resources, operational rules description, describes the process of operation, data requirements, relative to other process always confirm the single degree of weight vector for sorting

$$
\left(c_{1}, c_{2}, c_{3}, c_{4}, c_{5}\right), \quad c_{i}=\sum_{j=1}^{5} c_{i j} / \sum_{i=1}^{5} \sum_{j=1}^{5} c_{i j},
$$
the consistency of judgement matrix index relative consistency index for $\mathrm{CR}$, because $n=5$, so, according to the random consistency index RI table to get knowledge $R I=1.12$, by the formula $C R=\frac{C I}{R I}$. when $C R<0.10$, the thought model resources, operational rules description, describes the process of operation, data requirements, relative to other process always confirm the degree of consistency of judgment matrix is acceptable, otherwise the judgment matrix should be amended.

Calculate the Total Weight and Total Sorts to Levels. Rule layer is calculated above factors model of resources and operational rules description, describes the process of operation, data requirements, other process always confirm the degree of weight relative to the target layer, we finally to get the program in the fully available, the basic of the program is available, some available and unavailable for the target layer always confirm the degree of weight, and sorting through scheme selection.

First calculate the plan relative to the layer of the four criteria layer configuration of resources, operational rules description, describes the process of operation, data requirements, other process of single frequency weighting:

$$
\left(s_{1}^{k}, s_{2}^{k}, s_{3}^{k}, s_{4}^{k}\right), \quad s_{i}^{k}=\sum_{j=1}^{4} s_{i j}^{k} / \sum_{i=1}^{4} \sum_{j=1}^{4} s_{i j}^{k},
$$

Represent rule layer five element model resources, operational rules description, describes the process of operation, data requirements, other process, the values of 1,2 , $3,4,5$, here when model resources, operational rules description, describes the process of operation, data requirements, other process has nothing to do with the 
scheme of layer, then $s_{i}^{k}=0$. At last solution relative to the four target layer always confirm the total sorts of weight:

$$
\left(s_{1}, s_{2}, s_{3}, s_{4}\right), \quad s_{i}=\sum_{k=1}^{4} s_{i}^{k} c_{k}, k=1,2,3,4,5
$$

Table 2 confirm the total sorts of weight

\begin{tabular}{|c|c|c|c|c|c|c|c|}
\hline \multirow{7}{*}{ 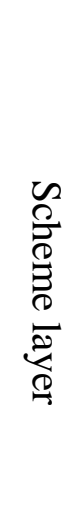 } & & \multicolumn{5}{|c|}{ Rule layer } & \multirow{3}{*}{ The total sequencing weight } \\
\hline & & $C_{1}$ & $C_{2}$ & $C_{3}$ & $C_{4}$ & $C_{5}$ & \\
\hline & & $c_{1}$ & $c_{2}$ & $c_{3}$ & $c_{4}$ & $c_{5}$ & \\
\hline & $S_{1}$ & $s_{1}^{1}$ & $s_{1}^{2}$ & $s_{1}^{3}$ & $s_{1}^{4}$ & $s_{1}^{5}$ & $s_{1}=\sum_{k=1}^{5} s_{1}^{k} c_{k}$ \\
\hline & $S_{2}$ & $s_{2}^{1}$ & $s_{2}^{2}$ & $s_{2}^{3}$ & $s_{2}^{4}$ & $s_{2}^{5}$ & $s_{2}=\sum_{k=1}^{5} s_{2}^{k} c_{k}$ \\
\hline & $S_{3}$ & $s_{3}^{1}$ & $s_{3}^{2}$ & $s_{3}^{3}$ & $s_{3}^{4}$ & $s_{3}^{5}$ & $s_{3}=\sum_{k=1}^{5} s_{3}^{k} c_{k}$ \\
\hline & $S_{4}$ & $s_{4}^{1}$ & $s_{4}^{2}$ & $s_{4}^{3}$ & $s_{4}^{4}$ & $s_{4}^{5}$ & $s_{4}=\sum_{k=1}^{5} s_{4}^{k} c_{k}$ \\
\hline
\end{tabular}

Expert Scoring and Calculating the Overall Aviation Anti-Submarine Military Conceptual Model Is Confirmed. Each expert according to their own judgment to the aviation anti-submarine military conceptual model, the basic use, part four scheme fully available, available, unavailable scores, the expert group for vector $\left(l_{1}, l_{2}, \cdots, l_{p}\right)$, the first expert grade four schemes for $\left(x_{1}^{k}, x_{2}^{k}, x_{3}^{k}, x_{4}^{k}\right)$, experts to the aviation anti-submarine warfare recognized degrees as military conceptual model:

$$
\sum_{m=1}^{4} x_{m}^{k} s_{m}
$$

An expert to the aviation anti-submarine warfare military conceptual model of total recognized degrees as:

$$
V=\sum_{k=1}^{p} \frac{1}{p}\left(\sum_{m=1}^{4} x_{m}^{k} s_{m}\right),
$$

It is assumed that the authority of experts, the same is weighted coefficient $\frac{1}{p}$.

If finally come to meet our requirements, it will eventually get the panel's final confirmation, otherwise will not be confirmed

\section{Example Analysis}

On the basis of the above discussion, we come to, for example, analyses about the aviation anti-submarine warfare experts confirm method of military conceptual model. First model resources, operational rules description, describes the process of operation, data requirements, other process relative to the total confirmation of a judgment matrix. 


$$
\left\{\begin{array}{ccccc}
1 & 1 / 2 & 1 / 4 & 1 / 6 & 1 / 8 \\
2 & 1 & 1 / 2 & 1 / 3 & 1 / 4 \\
4 & 2 & 1 & 1 / 2 & 1 / 2 \\
6 & 3 & 2 & 1 & 1 \\
8 & 4 & 2 & 1 & 1
\end{array}\right\}
$$

Consistency inspection, calculate $\lambda_{\max } \approx 5.4$ and $C R=0.089<0.10$ thus meet the requirements of the consistency of judgment matrix.

One sorting weight vector is: $(0.047,0.102,0.184,0.229,0.368)$;

structure the four schemes relative to the factors of judgement matrix in the rule layer:

(1) the first is the four schemes for judging matrix model resources, because the plan of the four "unavailable" resources "" model of rule layer is not related, so the weight $s_{4}^{1}=0$, and the other three schemes of judgment matrix for:

$$
\left\{\begin{array}{ccc}
1 & 1 / 2 & 1 / 4 \\
2 & 1 & 1 / 3 \\
4 & 3 & 1
\end{array}\right\}
$$

Four schemes for resources "model" single sorting weight for: $(0.134,0.254,0.612,0)$. layer

(2) by the same token, a calculation scheme of four other factors of the criterion

A process description of operations rules description, operations, data requirements, other process judgment matrix, respectively

$$
\left\{\begin{array}{ccc}
1 & 3 & 2 \\
1 / 3 & 1 & 2 \\
1 / 2 & 1 / 2 & 1
\end{array}\right\} 、\left\{\begin{array}{ccc}
1 & 4 & 2 \\
1 / 4 & 1 & 1 / 2 \\
1 / 2 & 2 & 1
\end{array}\right\} 、\left\{\begin{array}{ccc}
1 & 1 & 5 \\
1 & 1 & 5 \\
1 / 5 & 1 / 5 & 1
\end{array}\right\} 、\left\{\begin{array}{ccc}
1 & 3 & 5 \\
1 / 3 & 1 & 2 \\
1 / 5 & 1 / 2 & 1
\end{array}\right\}
$$

Calculate the single sorting weight as follows: $(0.529,0.294,0.117,0) 、(0.571$, $0.143,0,0.285)$

$(0.454,0,0.454,0.096) 、(0,0.641,0.237,0.122)$

Calculation scheme layer relative to the total confirmation of total order weight.

Table 3 rule and scheme

\begin{tabular}{|c|c|c|c|c|c|c|}
\hline \multirow{2}{*}{ Sule } & $C_{1}$ & $C_{2}$ & $C_{3}$ & $C_{4}$ & $C_{5}$ & $\begin{array}{c}\text { Sort } \\
\text { weight }\end{array}$ \\
\cline { 2 - 6 } & 0.047 & 0.102 & 0.184 & 0.229 & 0.368 & 0.26928 \\
6
\end{tabular}


Assuming that there are three experts' grade for each scheme are: $(8,5,6,5)$ 、

$(6,7,5,3) \quad(7,4,3,8)$

The three experts confirmed respectively: $5.653,5.254,4.744$

Total three experts for confirmation:

$$
V=\frac{1}{3}(5.653+5.254+4.744)=5.217
$$

Set the aviation anti-submarine warfare military conceptual model of total confirm degree greater than 5 think model is available, otherwise we can't use, so, for example, model is available.

\section{Conclusion}

This article only on aviation anti-submarine military conceptual model to confirm the preliminary discussion, there is no rule of four schemes for the plan layer of layer configuration of resources, operational rules description, describes the process of operation, data requirements, total sorts of other process consistency check, remains to be further research and deepening.

\section{References}

[1] Liwei L, Rongshuang F. Simulated annealing algorithm in solving frequency assignment problem[C]//Advanced Computer Theory and Engineering (ICACTE), 2010 3rd International Conference on. IEEE, 2010, 1: V1-361-V1-364.

[2] Castelino D J, Hurley S, Stephens N M. A tabu search algorithm for frequency assignment [J]. Annals of Operations Research, 1996, 63(2): 301-319.

[3] Alabau M, Idoumghar L, Schott R. New hybrid genetic algorithms for the frequency assignment problem [J]. Broadcasting, IEEE Transactions on, 2002, 48(1): 27-34.

[4] Radiom, S., Aliakbarian, H., etc., A Simple Real-Coded Compact Genetic Algorithm and its Application to Antenna Optimization, Microwave conference, APMC 2007.

[5] Francisco Herrera and Manuel Lozano. Gradual Distributed Real-Coded Genetic Algorithms [J]. IEEE transactions on evolutionary computation, 2000, 4(1):43-62.

[6] Díógenes Marcano and Filinto Durán. Synthesis of [7] Antenna Arrays Using Genetic Algorithms [J]. IEEE Antennas and Propagation Magazine, 42(3):12-20.

[7] Wen-Pin Liao, Fu-Lai Chu. Array pattern synthesis with null steering using genetic algorithm by controlling only the current amplitudes [J]. INT. J. ELECTRONICS, 1999, 86(4):445-457.

[8] Wen-Pin Liao, Fu-Lai Chu Application of Genetic Algorithms to Phase-Only Null Steering of Linear Arrays [J] Electromagnetics, 1997, 17:171-183.

[9] Fred Aust in, Michael lew is. Automated Maneuvering Decision for Air-to-Air Combat[R].AIAA-87-2393:659-669 